\title{
Regenerative light touch
}

\section{By Benjamin Boettner, Associate Editor}

Regenerative approaches to tooth repair often involve the use of invasive and complex procedures such as cell transplantation or the controlled delivery of growth factors. A Harvard University team believes that it has identified a simpler solution-low-power laser treatment-that can induce stem cell differentiation in dental tissue.

It remains to be seen whether the approach can restore functional dentin in human teeth and the extent to which it can induce differentiation in additional cell types.

Most common dental restoration procedures rely on the use of inert biocompatible materials, such as mineral composites combined with adhesive resins, the purpose of which are to directly replace the function of natural teeth. These biomaterials have been engineered to release factors that can induce the regeneration of dentin, which is a calcified tissue that is produced by specialized cells called odontoblasts that reside within tooth pulp.

For advanced procedures in which more substantial tissue regeneration is needed, such as dental implantation or periodontitis treatment, cell grafting techniques have been developed. However, placing the cells into the correct anatomical context and getting them to survive or differentiate has been challenging.

The precise mechanisms by which these methods can drive tooth regeneration are not clearly understood, but one key factor is transforming growth factor- $\beta 1$ (TGFB1), which has been shown to preserve adult tooth structure. ${ }^{2}$ It is deposited as a latent, inactive form in the extracellular environment; once it is activated, it drives the differentiation of stem cells. ${ }^{3,4}$

Now, a team at the Wyss Institute for Biologically Inspired Engineering at Harvard University has shown that even in the absence of any grafting material, low-power lasers can differentiate some dental stem cells into odontoblasts through the activation of TGFB1.

Low-power lasers had previously been anecdotally linked to stem cell regeneration in multiple cell types and also are commonly used in nonregenerative dental procedures like polymer cross-linking and tooth whitening. The Harvard team wanted to investigate whether the method could directly affect the function of dental stem cells.

The investigators first sought to test whether a low-power laser could improve tooth structure in rats with mechanically exposed tooth pulp. Rats whose exposed teeth were treated with a low-power laser for 5 minutes and then covered with a filling had greater production of tertiary dentin at 12 weeks than rats who did not receive light treatment.
To pinpoint the mechanism underlying dentin production, the team analyzed primary human dental stem cells exposed to a low-power laser. In cell culture, the laser light elevated levels of reactive oxygen species, which led to the activation of latent TGFB1. This release triggered a signaling cascade that induced the differentiation of mesenchymal stem cells into odontoblasts and promoted the production of tertiary dentin.

Blocking the cascade in vitro or in vivo using TGFB1 receptor inhibitors or using TGFB1 knockout mice prevented dentin formation, nailing down the requirement of TGFB1 for the light-induced effect.

Results were published in Science Translational Medicine. The team was led by David Mooney, a professor of bioengineering at the Harvard University School of Engineering and Applied Sciences and a core faculty member at the Wyss Institute.

Gianpaolo Papaccio, director of the Department of Experimental Medicine at the Second University of Naples, told SciBX, "These findings are of great interest because they represent a fundamental step in overcoming complications related to the grafting of stem cells. They provide a basis for future clinical treatment strategies."

Anibal Diogenes, an assistant professor of endodontics at The University of Texas Health Science Center at San Antonio, noted, "Dentists already use lasers for disinfection and curing dental materials. Thus, this technology is very likely to transfer easily to clinical practice. Hopefully, this technique will allow for better preservation of pulp vitality, increasing the predictability and success of direct and indirect pulp capping procedures following caries [cavity] removal."

\section{Tooth complex}

Although the ability of a low-power laser to drive stem cell differentiation is striking, the clinical utility of the approach will hinge on whether it can drive dentin formation in human teeth and direct the differentiation of additional types of dental stem cells at precisely targeted locations.

Frederic Michon, a team leader at the University of Helsinki's Institute of Biotechnology who studies dental stem cell populations, told SciBX that the usefulness of the approach might be limited to repairing damage located at the pulp-dentin interface.

"The method is focusing on dental pulp, which is specifically derived from mesenchymal stem cells. Other essential tooth tissues are generated by other stem cell lineages," he said. The effectiveness of low-power lasers in differentiating other cell lineages was not tested in the study.

Jeremy Mao, co-director of the Center for Craniofacial Regeneration at the Columbia University College of Dental Medicine, agreed that the method needs to be tested in other dental tissues but was optimistic it could be broadly effective. "Beyond punctured pulp, there might be a potential for laser activation of latent TGFB1 for other types of tooth regeneration, such as root formation," he said.

It also remains to be tested whether laser exposure will drive dentin formation in human cells. "Rat teeth are different from those in humans in that they are continuously regenerated. The translation to humans must necessarily first pass through in vitro experiments conducted on human primary dental cells," said Papaccio.

Although human dental stem cells were observed to switch on dentin-producing genes when exposed to a low-power laser, actual 
"Since we know that very low power has no effect while higher power has a detrimental response, outlining the therapeutic sweet spot is going to be key to translating this approach."

-Praveen Arany,

National Institutes of Health dentin deposition was only shown in rodent cell culture and teeth.

In addition, Papaccio said that the functionality of the dentin being produced remains in question. "The approach leads to tertiary dentin formation, which is usually produced by dental stem cells during tooth injury, and it is only organized in a sparse, irregular pattern that resembles the 'woven bone structure', which is not a true dentin; collagen fibers are arranged irregularly in the form of interlacing networks. Overall, it remains to be seen whether this technique can generate compact dentin resistant to mechanical stress."

Praveen Arany agreed. "Future attempts need to focus on organizing newly generated tissue in a normal, tubular pattern using better technique and perhaps additional cues." Arany is first author of the study and was at the Wyss Institute. He is now an assistant clinical investigator at the NIH.

Mooney added that the effect of low-power light could be further improved. "In our study we only treated once. Treating multiple times may enhance the effect and accelerate the repair process," he said.

Arany said that modulating the dose of the laser treatment could improve the method. "Since we know that very low power has no effect while higher power has a detrimental response, outlining the therapeutic sweet spot is going to be key to translating this approach." He is developing additional assays that can accurately monitor and predict the effect of low-power laser therapy.

Ophir Klein, an associate professor in the departments of orofacial sciences and pediatrics at the University of California, San Francisco, said that more exhaustive monitoring of pathways affected by laser exposure would be required to convince him of the safety of the approach and could be used to help optimize its efficacy.

"The study focused on a single pathway, and while it is clear that the effects are mediated to a large extent by TGFB1, it is likely that there are additional effects. Thus, an unbiased approach that examines a broader array of signals will be an important next step, as it is unlikely that the reactive oxygen species generated by the laser are exclusively targeting TGFB1." Klein is also an associate professor at the institutes for human genetics and regeneration medicine at UCSF.

A patent has been filed covering the method, and the IP is available for licensing from the Office of Technology Development at Harvard University.

Boettner, B. SciBX 7(26); doi:10.1038/scibx.2014.756

Published online July 10, 2014

\section{REFERENCES}

1. Arany, P.R. et al. Sci. Transl. Med.; published online May 28, 2014; doi:10.1126/scitranslmed.3008234

Contact: David J. Mooney, Harvard School of Engineering and Applied Sciences, Cambridge, Mass.

e-mail: mooneyd@seas.harvard.edu

2. D'Souza, R.N. et al. Eur. J. Oral Sci. 106, 185-191 (1998)

3. Discher, D.E. et al. Science 324, 1673-1677 (2010)

4. Mullen, A.C. et al. Cell 147, 565-576 (2011)

\section{COMPANIES AND INSTITUTIONS MENTIONED}

Columbia University College of Dental Medicine, New York, N.Y. Harvard University, Cambridge, Mass.

Harvard University School of Engineering and Applied

Sciences, Cambridge, Mass.

National Institutes of Health, Bethesda, Md.

Second University of Naples, Naples, Italy University of California, San Francisco, Calif.

University of Helsinki, Helsinki, Finland

The University of Texas Health Science Center at San Antonio, San Antonio, Texas

Wyss Institute for Biologically Inspired Engineering at Harvard University, Cambridge, Mass. 